\title{
Custo-efetividade de programa de educação para adultos asmáticos atendidos em hospital-escola de instituição pública*
}

\author{
Maria Alenita de Oliveira ${ }^{1}$, Maria Tereza Muniz², Lucia Ande Santos², \\ SONIA MARIA FARESIN ${ }^{1}$, ANA LUISA GODOY FERNANDES ${ }^{3}$
}

\begin{abstract}
Introdução: A asma pouco controlada acarreta custo econômico substancial para o sistema de saúde, principalmente, decorrente de gastos diretos. Programas de educação podem levar à redução desses custos. Objetivo: Avaliar os custos econômicos diretos resultantes da implantação de um programa de educação para asmáticos quando comparado com o atendimento usual especializado. Método:

Foram calculados, retrospectivamente, e comparados os custos diretos de um programa de educação $e$ do atendimento usual ao asmático. As hospitalizações, visitas ao pronto-socorro e visitas ambulatoriais, em ambos os grupos, foram registradas durante o período da intervenção educacional. Os valores utilizados para o cálculo desses custos foram baseados nos dados disponíveis no banco de dados do Datasus de 1996. O custo total com medicação/paciente no grupo educação (E) e controle (C) foi baseado no consumo de medicação durante o mês anterior à ultima visita e estimado pelo guia farmacêutico de fevereiro de 1996. Os valores finais foram convertidos em US dólar. Resultados: O custo médio direto em US dólar por paciente (US\$) dos grupos $\mathrm{C}$ e $\mathrm{E}$ e a diferença de custos $(\Delta)$ entre os dois foram, respectivamente: para hospitalizações - US $\$ 183$, US $\$ 0, \Delta=\$ 183$; visitas ao PS -

US $\$ 14$, US $\$ 5, \Delta=$ US $\$ 9$; visitas ambulatoriais agendadas - US $\$ 10$, US $\$ 24, \Delta=-U S \$ 14$; medicações - US $\$ 124,3$, US $\$ 195,6, \Delta=-$ US $\$ 71,3$. O custo total foi de US $\$ 331$ para o grupo C e US $\$ 224$ para o grupo E, o que permitiu redução de gastos da ordem de US\$107/paciente do grupo educação em relação ao controle. Conclusão: No grupo educação o custo total com medicamentos é

superior ao observado no grupo controle, refletindo maior gasto com medicação de prevenção.

Entretanto, a implantação do programa de educação leva a redução dos gastos com hospitalização e visitas ao PS, o que diminui os custos totais e torna o programa economicamente atrativo.
\end{abstract}

(J Pneumol 2002;28(2):71-76)

\section{Cost-effectiveness of an education program for asthmatic adults of a public university hospital}

The direct costs incurred in managing patients with poorly controlled asthma are high and educational programs could decrease these costs. Aim: The objectives of this study were to compare the direct cost of the implementation of an educational program for adult asthmatic patients with the cost of the usual care delivered to asthmatics by specialists. Methods: Five years ago, a six-month study demonstrated that an educational program improved clinical outcomes (22 in educational program-E and 20 patients in control group-C). Throughout the educational intervention period all cases of hospitalization, emergency and regular calls involving patients from both groups were recorded. The basis for the values utilized in the calculation of costs was the healthcare database of the Brazilian government (DATASUS). The overall medication

cost/patient in both groups was based on the amount of medication taken during the month preceding the last call. The final values were converted into US dollars. Results: The mean direct cost/patient in the educational $(E)$ and control $(C)$ groups and the difference $(\Delta)$ between groups were: hospitalizations ( $C=U S \$ 183, E=0, \Delta=U S \$ 183)$; emergency calls (C=US\$14,

* Trabalho realizado na Disciplina de Pneumologia da Universidade Federal de São Paulo (Unifesp).

1. Doutor em Medicina.

2. Estagiária da Disciplina de Pneumologia.

3. Professora Adjunto.
Endereço para correspondência - Maria Alenita de Oliveira, Disciplina de Pneumologia, Unifesp/EPM, Rua Botucatu, 740, $3^{\circ}$ andar 04023-004 - São Paulo, SP, Brasil. Fax (11) 5084-1268.

Recebido para publicação em 2/10/01. Aprovado, após revisão, em 10/12/01. 
$E=U S \$ 5, \Delta=U S \$ 9)$; regular calls $(C=U S \$ 10, E=U S \$ 24, \Delta=-U S \$ 14)$; medication $(C=$ US\$ 124,3, E = US\$195,6, $\Delta=-U S \$ 71,3)$. The total cost was US\$33/patient in group $C$ and US\$ 224/patient in group E with an average cost saving of US\$ 107/patient. Conclusion: The expenses with medication is higher in E group because the regular use of maintenance drugs, however the study suggested that the application of the asthma education program reduced the total direct costs of asthma.

Descritores - Asma. Análise custo-benefício. Educação do paciente.

Key words - Asthma. Cost-benefit. Patient education.

\section{INTRODUÇÃO}

A asma é uma doença crônica, cujo descontrole leva a freqüentes hospitalizações e visitas ao pronto-socorro (PS), eleva a morbidade e impõe grande impacto na vida social e econômica dos pacientes, piorando sua qualidade de vida. A educação do asmático é um dos pilares de sustentação do seu tratamento, que só será efetivo se baseado em medicação antiinflamatória regular.

A prevalência da asma no Brasil está entre uma das maiores do mundo, conforme dados encontrados no estudo ISAAC ${ }^{(1)}$. A asma foi a quarta causa de hospitalização em 1996, correspondendo a cerca de 350.000 internações em todo o país, a um custo de aproximadamente 76 milhões de reais. Isto equivale ao terceiro maior gasto do SUS com uma doença ${ }^{(2)}$. Os custo diretos com o tratamento da asma são calculados pelos gastos com medicamentos e aparelhos, consultas médicas e hospitalizações. Os custos indiretos envolvem absenteísmo à escola $e$ ao trabalho, tanto do paciente como do seu acompanhante, além de perda de produtividade, aposentadoria precoce e morte.

Em estudo prévio, demonstramos que a aplicação de um programa de educação em nosso meio leva a redução da morbidade da asma com redução das hospitalizações e visitas ao PS, além de melhora da qualidade de vida dos pacientes ${ }^{(3)}$. O presente estudo foi realizado para avaliar o custo econômico desse programa de educação para asmáticos comparado com um grupo de pacientes atendidos em ambulatório especializado e que receberam tratamento usual.

\section{MÉTODO}

Foram calculados, de forma retrospectiva, e comparados os custos diretos do programa de educação, implantado no ano de 1996, com os custos de um atendimento usual especializado.

Durante o cálculo do tamanho da amostra observou-se a necessidade de inclusão de 20 pacientes em cada grupo para avaliar a efetividade do programa de educação. $\mathrm{O}$
Siglas e abreviaturas utilizadas neste trabalho

Datasus - Sistema de Informações Hospitalares do SUS

E - Educação

$\mathrm{C}$ - Controle

PS - Pronto-socorro

ISAAC - International Study of Asthma and Allergies in Childhood

SUS - Sistema Único de Saúde

PFE - Pico do fluxo expiratório

$V_{E F}$ - Volume expirado forçado no primeiro segundo

cálculo foi realizado estimando-se redução de $50 \%$ nas variáveis de desfecho do grupo sob intervenção, considerando-se um erro alfa de 0,05 e o poder do teste de $80 \%$.

Foram recrutados, após sorteio, 80 portadores de asma moderada ou grave cadastrados no ambulatório de um hospital-escola pertencente a uma instituição pública (Unifesp), que recebe pacientes em situação socioeconômica desfavorável. Sessenta pacientes compareceram à entrevista e, após assinar o consentimento pós-informação, foram randomizados em grupo educação $(\mathrm{E})$ e controle (C).

\section{Programa de educação}

Os pacientes alocados no grupo educação foram submetidos a uma intervenção educacional estruturada, com monitorização da doença pelos sintomas respiratórios $e$ avaliação médica regular. Não foi fornecido nenhum plano de ação escrito. O programa educacional constava de seis avaliações mensais individuais e duas sessões em grupo.

As avaliações mensais incluíam pré-consulta, consulta médica propriamente dita e pós-consulta. Na pré-consulta foi mensurado o pico do fluxo expiratório, avaliada a habilidade para utilizar um nebulímetro de forma padronizada e o preenchimento de um diário de sintomas. A seguir, os pacientes receberam informações individualizadas sobre a doença, seu reconhecimento, profilaxia de fatores desencadeantes, diferença entre medicação de alivio e prevenção e sinais de descontrole da doença. $\mathrm{Na}$ pós-consulta foi revisada a prescrição médica e reforçado o preenchimento do diário.

Durante as sessões em grupo apresentou-se um vídeo produzido pelos investigadores, no qual se enfatizavam preconceitos com a asma, uso correto do nebulímetro, o 
tratamento baseado na identificação e redução de exposição aos fatores desencadeantes e identificação dos sinais de descontrole da doença. Ao término do vídeo realizava-se uma discussão entre os participantes para solucionar dúvidas e sanar dificuldades.

\section{Atendimento usual}

No atendimento usual o número de consultas foi determinado pelo médico que atendeu o doente de acordo com seu quadro clínico. Foram realizadas de duas a cinco consultas e a medicação prescrita foi baseada nas recomendações do I Consenso Americano de Asma ${ }^{(4)}$. Os pacientes não receberam instruções formais a respeito do controle da doença, não foram treinados para usar medicação dosimetrada e não preencheram diários, além de não participarem de sessões de discussão e apresentação de vídeos. Os pacientes receberam, somente, instruções verbais a respeito do uso adequado da medicação.

\section{Cálculo dos custos econômicos}

Foram levantadas as hospitalizações, visitas ao prontosocorro $e$ visitas ambulatoriais dos dois grupos durante os seis meses do estudo, para estimativa dos gastos diretos com a doença. Os valores utilizados para o cálculo foram baseados nos dados disponíveis no banco de dados do Datasus de $1996^{(5)}$. Segundo este, o custo médio de uma internação na região metropolitana da cidade de São Paulo foi estimado em 415,45 reais.

O custo médio de uma visita ao PS foi estimado somando-se o custo da consulta médica ao custo da medicação. O valor de 4,06 reais utilizado para a consulta médica no PS foi o mesmo da ambulatorial. Admitindo-se que um asmático ao ser avaliado no PS recebe, no mínimo, uma inalação (Atrovent, Berotec, soro fisiológico e oxigênio), cujo valor era de 3,20 reais, o custo mínimo estimado para uma visita ao PS foi de 7,26 reais.

Os valores de todas as medicações utilizadas pelos doentes em cada grupo, no último mês do estudo, foram somados. Este total foi dividido pelo número de pacientes do grupo, obtendo-se o custo médio mensal/paciente. Multiplicando-se este valor por seis foi obtido o custo total com medicação/paciente nos grupos E e C. Para esse cálculo foi utilizado como referência o guia farmacêutico de fevereiro de $1996^{(6)}$.

Os valores em reais foram convertidos em US dólar para atualização monetária, considerando-se 1 dólar equivalente a 0,9806 reais, segundo dados fornecidos pelo Banco Central e correspondentes a $13 / 2 / 1996^{(7)}$.

O cálculo dos custos basais foi efetuado para ambos os grupos seis meses antes do início do estudo, porém, como não havia dados sobre o número de visitas ambulatoriais, o custo final foi efetuado sem este valor. Por esse motivo, não pudemos comparar os custos considerando seis meses antes e ao término da intervenção educacional. Só foi possível comparar os gastos no início e final da intervenção.

\section{Resultados}

Os dados demográficos dos dois grupos estão listados na Tabela 1.

Quando comparamos os dados do grupo controle com o grupo educação, no inicio da intervenção, baseados nos dados referentes aos seis meses anteriores, observamos que os doentes apresentavam custos equivalentes em relação ao número de visitas ao PS e medicamentos utilizados (Tabela 2). O custo com hospitalizações foi maior no grupo controle do que no grupo educação antes de iniciar o programa educacional, mas esta diferença não foi

TABELA 1

Dados demográficos do grupo controle e educação

\begin{tabular}{|c|c|c|}
\hline & $\begin{array}{c}\text { Educação } \\
\text { n = 22 }\end{array}$ & $\begin{array}{c}\text { Controle } \\
n=20\end{array}$ \\
\hline Sexo F/M & $20 / 2$ & $17 / 3$ \\
\hline Idade $(\bar{x} \pm D P)$ & $41 \pm 15$ & $38 \pm 17$ \\
\hline Asma grave $(\%)$ & 36 & 35 \\
\hline Asma moderada (\%) & 64 & 65 \\
\hline Co-morbidade (\%) & 50 & 50 \\
\hline VEF $_{1}$ pré-BD em \% prev ( $\left.\bar{x} \pm D P\right)$ & $70 \pm 22$ & $80 \pm 19$ \\
\hline Renda mensal em US\$ ( $\bar{x} \pm D P)$ & $486 \pm 394$ & $413 \pm 233$ \\
\hline Escolaridade (anos) & $5 \pm 4$ & $5 \pm 3$ \\
\hline Analfabeto $(\%)$ & 9 & 10 \\
\hline$<5$ anos de educação (\%) & 72 & 60 \\
\hline > 5 anos de educação (\%) & 18 & 30 \\
\hline
\end{tabular}

TABELA 2

Custo em US\$ dos parâmetros para o grupo educação e grupo no início e término de uma intervenção educacional estruturada

\begin{tabular}{lcccccc}
\hline & \multicolumn{2}{c}{ Educação } & & \multicolumn{2}{c}{ Controle } \\
\multicolumn{1}{c}{ Custo em US\$ } & Antes & Depois & & Antes & Depois \\
\hline \multirow{2}{*}{ Hospitalizações } & 148,0 & 0 & & 285,1 & 183 \\
Visitas ao PS & 26,8 & 5,0 & & 27,3 & 14,0 \\
Visitas ambulatoriais & nd & 24,0 & & nd & 10,0 \\
M edicações* & 122,2 & 195,6 & & 124,8 & 124,3 \\
Corticóide inalatório & 42,0 & 104,6 & & 57,7 & 51,7 \\
Corticóide oral & 31,7 & 52,4 & & 30,0 & 34,7 \\
$\quad$ Xantinas & 28,6 & 26,5 & & 17,1 & 25,3 \\
B2-agonista de curta duração & 19,9 & 12,1 & & 20,2 & 12,6 \\
\hline
\end{tabular}

* custo médio dos medicamentos/doente 
estatisticamente significante. Os custos com hospitalizações corresponderam a $65,2 \%$ e $48,3 \%$ dos custos totais, respectivamente, nos grupos $\mathrm{C}$ e $\mathrm{E}$ (Tabela 2).

Antes da intervenção foram obtidos os gastos médios de US\$ 297,0 no grupo educação e US\$ 437,4 no grupo controle. Na Tabela 2 encontram-se, também, os custos parciais no grupo educação e controle ao final da intervenção educacional.

Ao final da intervenção educacional, como nenhum doente do grupo educação foi hospitalizado, verificou-se redução de custos de $100 \%$ neste item (US\$ 148; US\$ $0)$. No grupo controle os custos com hospitalização sofreram redução de $36 \%$, pois caiu de US $\$ 285,1$ para US $\$$ 183.

Analisando-se os custos com visitas ao PS, observou-se que no grupo educação houve redução de $81,3 \%$, pois caiu de US\$ 26,8 para US\$ $\$$. No grupo controle o valor inicial de US\$27,3 decresceu para US\$14, conferindo redução de $48,7 \%$.

A comparação de custos com visitas ambulatoriais não pôde ser efetuada, pois não conseguimos calcular os valores nos seis meses antes do início do programa.

Quando analisamos os gastos com medicações, observamos que no início do estudo não houve diferença entre os dois grupos, mas ao término, no grupo controle os gastos permaneceram iguais (US\$ 124,8 e US\$ 124,3), e no grupo educação houve aumento de custos de $60,1 \%$ (US\$122,2 e US\$ 195,6) (Tabela 2).

\section{DISCUSSÃO}

A análise econômica de qualquer intervenção, seja ela medicamentosa ou não, só tem sentido se essa intervenção é eficaz.

A revisão sistemática realizada pela Cochrane Library ${ }^{(8)}$ e publicada em 2001 conseguiu reunir 58 estudos que tratavam de programas de educação aplicados em adultos portadores de asma. Desses, 29 estudos foram incluídos na análise final, sendo 25 ensaios clínicos controlados e randomizados, o que assegura o bom nível de evidência científica dos resultados aferidos.

Ao final da revisão havia 4.382 pacientes randomizados e 2.856 completaram o estudo, possibilitando concluir que a intervenção educacional leva a melhores desfechos de saúde, ou seja, permite melhor controle da asma, diminui o número de hospitalizações, visitas ao PS, visitas não agendadas ao ambulatório, absenteísmo ao trabalho e à escola e episódios de asma noturna. Esses efeitos são suficientemente grandes para alcançar significância estatística e clínica.

Os programas que levam aos melhores resultados são estruturados e em geral se utilizam da forma verbal, escrita, visual e/ou auditiva para transmitir conhecimentos.
Incluem um plano de ação escrito, monitorização dos sintomas e revisão médica regular. Programas com essas características deveriam ser oferecidos aos adultos portadores de asma. A monitorização da doença pode ser realizada através de escore de sintomas ou pela mensuração do pico do fluxo expiratório (PFE). A longo prazo, a mensuração do pico de fluxo leva à diminuição de adesão. Em trabalho recente, Côté et al. ${ }^{(9)}$ observaram que somente $30 \%$ dos pacientes suportam monitorizar o PFE por mais de um ano. O plano de ação pode ser colocado em prática pelo próprio paciente (automanejo) ou pelo seu médico. As intervenções menos intensivas, sem plano de ação escrito, são menos eficazes.

Diante dessas conclusões, resta saber se economicamente esses programas são viáveis. A análise econômica ideal deveria comparar todos os programas existentes, incluir os benefícios e os custos diretos, indiretos $e$ os intangíveis, estes últimos, na verdade, inacessíveis.

Não há na literatura número substancial de estudos que enfoquem tal objetivo e, dos existentes, nenhum foi planejado para ser primariamente um estudo de análise econômica.

Em geral, trata-se de ensaios clínicos que se preocupam com o que é melhor para o paciente, ao passo que os estudos de análise econômica apresentam uma perspectiva social. A efetividade clínica é necessária para que a terapia seja avaliada economicamente, mas o tratamento pode ser clinicamente efetivo e, ainda assim, economicamente inviável. Por exemplo, seu alto custo pode impossibilitar sua execução em grande escala(10).

Análises de custo econômico englobam vários métodos para avaliar e quantificar os custos, as conseqüências clínicas dos tratamentos e cuidados médicos, e estimar o valor econômico da intervenção em relação aos tratamentos alternativos ou ao não tratamento.

O melhor estudo de análise econômica é o de custoefetividade, no qual se utilizam medidas de desfechos relacionadas à história natural da doença. Nesse caso, seria avaliar o custo de uma exacerbação de asma abortada, de uma hospitalização evitada, de uma morte prevenida e, mais recentemente, os dias livres de sintomas da doença ${ }^{(11,12)}$.

Em nossa instituição, há vários anos se desenvolveu um programa de educação estruturado e dirigido para adultos portadores de asma. O conhecimento foi transmitido de forma verbal, visual e auditiva e incluía a monitorização da doença pelos sintomas, uso de broncodilatador de alívio e mensuração do PFE, além de contar com visitas médicas regulares, nos melhores moldes descritos pela revisão sistemática ${ }^{(3,13)}$.

Mesmo sem um plano de ação escrito, ficou provado que pacientes do grupo educação apresentaram menor número de hospitalizações, visitas ao PS, visitas não agen- 
dadas ao ambulatório e redução dos sintomas, quando comparados com um grupo de pacientes que receberam tratamento usual. Esses resultados parecem estar relacionados à baixa condição socioeconômico-cultural da nossa população, que permite evidenciar de maneira contundente a mudança de comportamento e a aquisição de habilidades provocadas pela intervenção educacional. Em populações com alto padrão socioeconômico-cultural os programas de educação pouco acrescentariam aos seus participantes.

Este trabalho consiste em um estudo de custo-efetividade derivado de um estudo anterior que mensurou apenas a efetividade do programa ${ }^{(3,13)}$.

Os custos médicos diretos decorrentes do tratamento da asma representam cerca de $52 \%$ dos gastos nos Estados Unidos. Desses gastos diretos, aproximadamente 53\% são decorrentes de custos hospitalares e visitas ao $\mathrm{PS}^{(14)}$. No nosso estudo, antes da intervenção educacional observamos que os custos com hospitalização alcançaram $48,3 \%$ no grupo $\mathrm{E}$ e $65,2 \%$ no grupo $\mathrm{C}$, em relação aos gastos totais.

O presente estudo incluiu portadores de asma moderada e grave, o que favorece a análise econômica, pois a magnitude dos custos encontra-se relacionada com a gravidade da doença. Estima-se que os custos anuais com asma leve sejam de US\$ 76 por paciente e US\$ 13.572 por paciente com asma grave ${ }^{(15)}$. Dessa forma, os doentes com asma grave (cerca de $10 \%$ dos doentes asmáticos) são responsáveis pela maior proporção de gastos com a doença.

Após a intervenção educacional observouse redução dos custos com hospitalizações e visitas ao PS e aumento dos gastos com medicação.

Infelizmente, como em nosso meio não há fornecimento de medicação antiinflamatória, essencial para o controle da asma moderada e grave, o custo da estabilidade da doença tem profundo impacto sobre a renda familiar dos doentes. Nessa população a renda mensal média foi de US\$ 486 para o grupo E e US\$ 413 para o grupo C. O custo médio estimado com medicação foi de US $\$ 32$ mensais, o que equivaleu a $7 \%$ da renda dos nossos doentes.

Os resultados dos estudos de custo-efetividade não são unânimes. Há poucos estudos direcionados para adultos e o melhor estudo, com essa característica, foi realizado em crianças. Na Tabela 3 documentamos três estudos de custo-efetividade, referendados na literatura, acompanhados pelos resultados encontrados no nosso trabalho $e$ demonstrativos da redução de custos proporcionada pelas intervenções educacionais ${ }^{(16-18)}$.

Estudos conduzidos na Finlândia, entretanto, apresentaram resultados contraditórios, pois uma relação de custo-efetividade de 118 marcos por dia de saúde ganho não foi conseguida em estudo posterior ${ }^{(19,20)}$.

O estudo de Sondeergaard et al. ${ }^{(21)}$ que estimou os custos e benefícios, em 62 adultos e crianças, de uma intervenção educacional por seis meses, também, evidenciou uma relação inapropriada, pois o custo total da intervenção foi de 6.546 libras e o benefício, de 4.528 libras.

Nosso trabalho evidenciou redução de gastos de $33 \%$ em relação ao atendimento usual especializado. Cifras

TABELA 3

Estudos de custo-efetividade envolvendo intervenção educacional associada ao tratamento de asma

\begin{tabular}{|c|c|c|c|c|}
\hline & Clark et al. & Windsor et al. & Balton et al. & Oliveira et al. \\
\hline População & Crianças & Adultos & Adultos & Adultos \\
\hline Local & $\begin{array}{l}\text { Clínica pediátrica } \\
\text { de alergia }\end{array}$ & $\begin{array}{l}\text { Clínica } \\
\text { pneumológica }\end{array}$ & $\begin{array}{l}\text { Moradores zona } \\
\text { rural e urbana }\end{array}$ & $\begin{array}{l}\text { Hospital } \\
\text { universitário }\end{array}$ \\
\hline Cidade & Nova York & Birmingham & & São Paulo \\
\hline Estudo & $\begin{array}{l}\text { Prospectivo, } \\
\text { randomizado } \\
(2: 1) \text {, controlado }\end{array}$ & $\begin{array}{l}\text { Prospectivo, } \\
\text { randomizado, } \\
\text { controlado }\end{array}$ & $\begin{array}{l}\text { Prospectivo, } \\
\text { randomizado, } \\
\text { controlado }\end{array}$ & $\begin{array}{l}\text { Prospectivo, } \\
\text { randomizado, } \\
\text { controlado }\end{array}$ \\
\hline Número & 310 & 267 & 241 & 42 \\
\hline Duração & 1 ano & 1 ano & 1 ano & 6 meses \\
\hline Intervenção & $\begin{array}{l}6 \text { sessões } 1 \text { h/cada } \\
5 \text { sessões adultos e } \\
\text { crianças separados }\end{array}$ & $\begin{array}{l}1 \text { sessão paciente } \\
1 \text { sessão peq. grupo } \\
2 \text { telefonemas }\end{array}$ & $\begin{array}{l}3 \text { sessões peq. } \\
\text { grupos }\end{array}$ & $\begin{array}{l}6 \text { sessões/paciente } \\
2 \text { sessões peq. } \\
\text { grupos }\end{array}$ \\
\hline Aplicador & Educador & Educador & Enfermeira & Educador \\
\hline Controle & Tratamento usual & $\begin{array}{l}1 \text { curta sessão } \\
2 \text { contatos }\end{array}$ & Tratamento usual & Tratamento usual \\
\hline Custo & $\begin{array}{l}\text { Poupa } \$ 0,62 / \$ 1,00 \\
\text { Grupo todo } \\
\text { Poupa } \$ 11,22 / \$ 1,00 \\
\text { Grupo alto risco }\end{array}$ & $\$ 28,42 /$ paciente & $\begin{array}{l}\text { \$ 543/paciente } \\
\$ 82 \text { educação } \\
\text { \$ } 628 \text { visitas PS }\end{array}$ & $\begin{array}{l}\text { Poupa US\$ 107/ } \\
\text { paciente }\end{array}$ \\
\hline Efeitos de saúde & $\begin{array}{l}\text { Sem } \neq \text { no grupo todo } \\
\downarrow 52 \% \text { hospital } \\
\downarrow 53 \% \text { visitas PS }\end{array}$ & $\begin{array}{l}\text { Escore de adesão } \\
\uparrow 44 \% \text { intervenção } \\
\uparrow 2 \% \text { controle }\end{array}$ & $\begin{array}{l}\downarrow \text { visitas ao PS } \\
\downarrow \text { dias limitação } \\
\text { G rupo intervenção }\end{array}$ & $\begin{array}{l}\downarrow \text { visitas ao PS } \\
\downarrow \text { escore de } \\
\text { sintomas }\end{array}$ \\
\hline
\end{tabular}


semelhantes foram observadas na Índia, onde Ghosh et al. ${ }^{(22)}$ evidenciaram melhora do pico de fluxo expiratório e redução do número de dias perdidos no trabalho associados a redução de custos de $22 \%{ }^{(13)}$.

É provável que esses diferentes resultados decorram do tipo de população que fez parte do programa educacional. Clark et al. ${ }^{(16)}$ conseguiram documentar essa possibilidade. Avaliando todo o grupo, observaram que não havia melhora nas medidas de desfechos avaliadas, mais precisamente, hospitalizações e visitas ao PS, o que conferiu péssima relação de custo-efetividade, ou seja, para cada US\$ 0,62 poupados foram gastos US\$1,00 com educação. Essa relação melhorou muito quando os autores analisaram somente pacientes que procuravam assiduamente os serviços de saúde, verificando que houve redução de $52 \%$ nas hospitalizações e $53 \%$ nas visitas ao PS, o que proporcionou poupança de US $\$ 11,22$ para cada US $\$ 1,00$ gasto com educação.

Assim, a implantação de programas de educação para doentes selecionados em países em desenvolvimento seria uma forma de reduzir os índices de morbidade e direcionar recursos sempre tão escassos.

Como em todo estudo retrospectivo, algumas limitações devem ser levadas em consideração. Os valores atribuídos ao número de visitas ao PS estão subestimados, já que não computamos os gastos com medicação endovenosa, utilização de equipamentos e exames efetuados. Os custos indiretos, também, não foram computados, o que aumentaria a relação custo-benefício da intervenção em relação ao controle. Esses custos em geral envolvem perda de emprego, perda de direção da casa, absenteísmo ao trabalho e escola, mortalidade, gastos com transporte para receber atendimento médico e tempo de espera para $o$ atendimento.

A nossa proposta foi comparar o impacto das duas formas de manejo no custo direto, considerando que o nosso programa foi direcionado para grupos pequenos de doentes para avaliar sua viabilidade e eficácia. Se considerarmos o aumento dos doentes em cada grupo, haveria relação custo-benefício maior.

A maior adesão aos corticóides inalatórios no grupo educação foi o responsável pelo aumento dos gastos neste grupo. Os corticóides consomem cerca de $54 \%$ dos gastos com medicação (Tabela 4) e os custos com medicamentos representam percentual importante da renda familiar, para que doentes asmáticos permaneçam com a doença estável.

Durante a intervenção, observamos diminuição dos custos de US\$107/doente/seis meses no grupo educação em relação ao grupo controle, a despeito do aumento de gastos com medicação e visitas ambulatoriais. Essa diminuição é atribuída à grande redução das hospitalizações verificadas no grupo educação.
Portanto, a relação custo-efetividade deste programa de educação para adultos asmáticos foi muito positiva $e$ torna-o economicamente atrativo.

\section{REFERÊNCIAS}

1. International Study of Asthma and Allergy in Childhood Worldwide. Variations in the prevalence of asthma symptoms: International Study of Asthma and Allergies in Childhood (ISAAC). Eur Respir J 1998;12: 315-35.

2. Sociedade Brasileira de Pneumologia e Tisiologia, Sociedade Brasileira de Alergia e Imunopatologia, Sociedade Brasileira de Pediatria. II Consenso Brasileiro no Manejo da Asma. J Pneumol 1998;24:171-276.

3. De Oliveira MA, Faresin SM, Bruno VF, Bittencourt AR, Fernandes ALG. Evaluation of an educational programme for socially deprived asthma patients. Eur Respir J 1999;14:1-7.

4. NHLBI. National Asthma Education Program, Expert Panel Report. Guidelines for the diagnosis and management of asthma. J Allergy Clin Immunol 1991;88:425.

5. Datasus. Sistema de Informações Hospitalares do SUS. Available from: htpp://www.datasus.gov.br/cgi/idb97/recur/e12.htm.

6. Guia farmacêutico Brasíndice, ano XXXI, nº 363-2 de janeiro de 1996.6

7. Homepage do Banco Central do Brasil. Séries Históricas. Available from: http://www. bcb.gov.br.

8. Gibson PG, Couglan J, Wilson AJ, Abramson M, Bauman A, Hensley MJ, Walters EH. Self-management education and regular practitioner review for adults with asthma (Cochrane Review). In: The Cochrane Library, Issue 2. Oxford: Update Software, 2001.

9. Côté J, Bowie DM, Robichaud P, Parent J-G, Battisti L, Boulet L-P. Evaluation of two different educational interventions for adult patients consulting with an acute asthma exacerbation. Am J Respir Crit Care Med 2001;163: 1415-9.

10. Krahn M. Issues in the cost-effectiveness of asthma education. Chest 1994;106:264S-9S.

11. Sullivan SD, Weiss KB. Health economics of asthma and rhinitis. II. Assessing the value of interventions. J Allergy Clin Immunol 2001; 107:203-10.

12. Sullivan S, Elixhauser A, Buist S, Luce BR, Eisenberg J, Weiss KB. National asthma education and prevention program working group report on the cost effectiveness of asthma care. Am J Respir Crit Care Med 1996;154:S84S95.

13. Oliveira MA, Bruno VF, Ballini LS, Jardim JRB, Fernandes ALG. Evaluation of an educational program for asthma control in adults. J Asthma 1997; 34:395-403

14. Weiss KB, Gergen PJ, Hodgsar TA. An economic evaluation of asthma in the United States. N Engl J Med 1992;326:862-6.

15. Buist AS. Cost-effectiveness of asthma management strategies. Eur Respir Rev 1995;5:292-4

16. Clark NM, Feldman CH, Evans D, Levison MJ, Wasilewski Y, Mellins RB. The impact of health education on frequency and cost of health care use by low income children with asthma. J Allergy Clin Immunol 1986;78:10815 .

17. Windsor RA, Bailey WC, Richards JM, Manzella B, Soong S-J, Brooks M. Evaluation of the efficacy and cost effectiveness of health education methods to increase medication adherence among adults with asthma. Am J Public Health 1990;80:1519-21.

18. Bolton MB, Tilley BC, Kuder J. The cost and effectiveness of an education program for adults who have asthma. J Gen Intern Med 1991; 6:401-7.

19. Laahdensuo A, Haahtela T, Herrala J, Kava T, Kiviranta K, Kuusisto P. Randomized comparison of cost-effectiveness of guided self-management and traditional treatment of asthma in Finland. BMJ 1998;316: 1138-9.

20. Kauppinen R, Sintonen H, Tukiainen H. One-year economic evaluation of intensive vs conventional patient education and supervision for self-management of new asthmatic patients. Respir Med 1998; 92: 300-7.

21. Sondeergaard B, Davidsen F, Kirkeby B, Rasmussen M, Hey H. The economics of an intensive education programme for asthmatic patients: a prospective controlled trial. Pharmacoeconomics 1992;1:207-12.

22. Ghosh CS, Ravindran P, Joshi M, Stea SC. Reductions in hospital use from self-management training for chronic asthmatics. Soc Sci Med 1998;46:1087-93. 